\title{
Dietary fibre, nuts and cardiovascular diseases
}

\author{
Jordi Salas-Salvadó ${ }^{1 *}$, Mónica Bulló ${ }^{1}$, Ana Pérez-Heras ${ }^{2}$ and Emilio Ros ${ }^{2}$ \\ ${ }^{1}$ Unitat de Nutrició Humana, Facultat de Medicina i Ciències de la Salut de Reus, Universitat Rovira i Virgili, Reus, Spain \\ ${ }^{2}$ Unitat de Lípids, Sevei d'Endocrinologia i Nutrició, Institut d'Investigacions Biomèdiques August Pi Sunyer, \\ Hospital Clínic, Barcelona, Spain
}

\begin{abstract}
Dietary fibre has a range of metabolic health benefits. Through a variety of mechanisms, dietary fibre, and the viscous variety in particular, slows down gastric emptying and intestinal transit, decreases the rate of intestinal carbohydrate absorption, and increases faecal bile acid excretion. Therefore, consumption of some types of soluble fibre can enhance satiety, which is associated with a lower BMI, and reduce blood cholesterol and the postprandial glucose response. Surprisingly, the consumption of insoluble fibre from whole grains, though metabolically inert, has been associated with a reduction in the risk of developing coronary heart disease and diabetes in epidemiological studies. The likely reason is that whole grains, like nuts, legumes and other edible seeds, contain many bioactive phytochemicals and various antioxidants. After cereals, nuts are the vegetable foods that are richest in fibre, which may partly explain their benefit on the lipid profile and cardiovascular health.
\end{abstract}

After decades of clinical and epidemiological research, there is a general consensus about the overall dietary pattern which is best associated with good cardiovascular health. This pattern consists of a high intake of plant foods (wholegrain cereals, vegetables, legumes, fruit and nuts) and fish, habitual use of unsaturated oils and fats, a low intake of animal fats (including meat and meat products, and enriched dairies) and manufactured foods rich in fat and/or simple sugars (including sweetened drinks), and moderate drinking of alcoholic beverages with meals (Hu \& Willett, 2002).

Dietary fibre, an indigestible vegetable residue, is one of the most frequently studied dietary components because of its cardiovascular protective effects. We will briefly review the beneficial properties of fibre for cardiovascular health in general and in the context of its intake with nuts, a fibre-rich food.

\section{Carbohydrates and dietary fibre}

The main sources of carbohydrates in the diet are foods of plant origin: cereals, vegetables, legumes and fruit. A distinction should be made between the slowly absorbed complex carbohydrates (starch) contained in natural vegetables, often associated with indigestible polymers (dietary fibre), and the rapidly absorbed simple carbohydrates (sugars) contained in milk (lactose), fruit (fructose) and table sugar (sucrose). Simple carbohydrates would also include numerous dietary products, such as pastries, cakes and chocolates, sweets and various sweetened drinks, foods that are so often consumed in contemporary diets. The distinction between the two types of carbohydrates is an important one because, when they substitute total fat, complex carbohydrates often have beneficial effects on the lipid profile and glucose metabolism. Simple carbohydrates induce hypertriglyceridemia and hyperinsulinism, and favour the development of obesity and diabetes in predisposed people (Reaven, 1997; Parks \& Hellerstein, 2000). When the proportion of fat in the diet is reduced, the consumption of carbohydrate increases since the consumption of proteins remains relatively constant. Carbohydrate-rich diets ( $\geq 60 \%$ En) that contain simple sugars are associated with increased triglyceride levels, reduced HDL cholesterol and a poor metabolic control of diabetes. However, when substituting complex carbohydrates for fat in the diet, both the lipid profile and glucose control are improved, and these effects may be ascribed in part to dietary fibre (Parks \& Hellerstein, 2000).

Dietary fibre, made up of indigestible carbohydrates and lignin which can be found whole in plants, is an important component of a vegetable-rich diet. Its effects on digestive physiology, lipid metabolism, glucose homeostasis and general health have raised considerable interest since 1974, when Burkitt et al. (1974) formulated the hypothesis that a low consumption of fibre in Western societies was one cause underlying the increased incidence of a variety of diseases linked to progress. Among these are obesity, diabetes, cholelithiasis, coronary heart disease (CHD), diverticular disease of the colon and colorectal cancer.

\section{Types of dietary fibre}

There are two types of dietary fibre, defined by their physical behaviour in water: insoluble fibre, such as cellulose, lignin and some hemicelluloses, which are abundant in wholegrain cereals (wheat bran is particularly rich in this type of fibre), and soluble fibre, such as gums, mucilages and pectins, contained specially in fresh vegetables, legumes and fruit, and the beta-glucans, present in oats, barley and some yeasts. One particular variety of soluble fibre is psyllium, a hydrophilic colloid that is found in the bark of the ispaghula husk and is used as a non-cathartic laxative, because it increases faecal 
mass, and as a cholesterol-lowering agent in patients with moderate hypercholesterolemia (Anderson et al. 2000). Also classifiable as soluble fibres are the non-digestible shortchain polysaccharides known as fructo-oligosaccharides or fructans, the main examples of which are inulin and oligofructose (Roberfroid \& Delzenne, 1998).

Most vegetable products contain a mixture of soluble and insoluble fibre in a proportion of approximately 1:3. Table 1 shows a list of plant foods and their total fibre content (Marlett, 1992). The foods that are richest in fibre are whole cereals, followed by nuts and legumes. The fibre content of these three food products is greater than that of refined cereals, fruits and fresh vegetables. Although all food contains both soluble and insoluble fibre, oats (and particularly oat bran), barley, legumes, apples and citrus fruits contain the highest quantities of soluble fibre, while wheat bran, bread and wholegrain cereals contain greater quantities of insoluble fibre. As shown in Fig. 1, the content of total fibre in nuts is high (USDA, 1984; Holland et al. 1991). The nut content of soluble fibre is appreciable, although most is insoluble (Marlett, 1992). Fresh vegetables, cereals and fruits are the main sources of fibre in our diet. Bearing in mind that nuts contribute little to total caloric intake in industrialized countries, the amount of fibre they contribute to the diet is considerable (Arija et al. 1996).

Different plant foods have different amounts and types of fibre. Whole wheat flour, bran and vegetables are, in general, good sources of cellulose, while bran and wholegrain cereals contain sizeable amounts of hemicellulose. Lignin is mainly found in ripe vegetables, wheat and some edible seeded fruits, such as strawberries. The total lignin content of the diet is very low, approximately $1 \mathrm{~g} / \mathrm{d}$, and mainly comes from wheat and fruit, vegetables with skin, and/or edible seeds. Oats, barley and legumes (from which 'guar' is derived) are rich in gum, while apples, citrus fruits, strawberries and carrots contain appreciable amounts of pectin. There is little difference in the content of fibre of raw and cooked fruits or vegetables, although its quality and structure may vary. Nuts are particularly rich in cellulose, followed by hemicellulose, pectin and lignin (Marlett, 1992).

\section{Metabolic effects of fibre}

Because it is indigestible in the human intestine and is made up of long-chain polymers that retain water, fibre in general, and insoluble fibre in particular, reduces intestinal transit

Table 1. Fibre content of selected foods and food groups

\begin{tabular}{lc}
\hline & $\mathrm{g} / 100 \mathrm{~g}$ \\
\hline Wholegrain cereals & $15-30$ \\
Nuts & $5-10$ \\
Legumes & $3-6$ \\
Wholemeal bread & 5 \\
Raisins & 4 \\
White bread & $2-3 \cdot 5$ \\
Leaf vegetables and fruit & $1.5-4$ \\
Potatoes and other tubers & $1-3$ \\
Vegetables & $0.5-3$ \\
White rice and pasta & $0.5-2$ \\
\hline
\end{tabular}

Taken from Marlett, 1992. time and increases the hydration of the faecal bolus, thus acting as a physiological laxative. The metabolic effects of fibre (reduction of blood cholesterol and postprandial glucose peaks) can be attributed solely to soluble fibre and, more specifically, to viscous fibre (Jenkins et al. 2000), these effects are summarized in Fig. 2. Viscosity is the ability to form gels. Because it is well-hydrated, this type of fibre can make the intestinal contents more viscous, thereby reducing the rate of absorption and spreading the nutrient load over time. Factors involved include resistance to bulk diffusion due to the increased viscosity of the luminal contents and increased resistance of the unstirred water layer that lines the absorptive surface of enterocytes. Because the distal ileum is the absorptive site for bile acids, increasing viscosity as water is progressively removed from the luminal contents will hamper bile acid absorption. This effect, together with physical binding of bile acids to the fibre, causes increased faecal bile acid loss and is the principle mechanism by which fibre may reduce serum cholesterol.

Nevertheless, the hypocholesterolemic effect of soluble fibre is modest, as the decrease in total and LDL cholesterol has been estimated to be around $0.05 \mathrm{mmol} / \mathrm{l}$ for every gram of soluble fibre added to the diet (Brown et al. 1999). For example, eating two apples a day contributes $2 \mathrm{~g}$ of pectin to the diet, which can reduce blood cholesterol by $0.10 \mathrm{mmol} / \mathrm{l}$ during the period of consumption. Likewise, the intake of $10 \mathrm{~g}$ psyllium a day as a supplement in people with moderate hypercholesterolemia reduces LDL cholesterol by an average of $7 \%$, which is already an appreciable figure (Anderson et al. 2000).

It has been known for some time that the intake of soluble fibre, such as guar gum (obtained from legumes, such as beans) and pectin, modifies the absorption kinetics of glucose, reducing the postprandial peaks of glucose and insulin (Jenkins et al. 1976; Potter et al. 1981). Its intake at meal times, therefore, can be a useful adjunct in the treatment of diabetes (Chandalia et al. 2000). The reduction of blood glucose is also related to viscosity causing a delay in gastric emptying. This, together with the decreased bulk diffusion due to both intraluminal trapping and increased resistance of the unstirred water layer, results in lower postprandial glucose peaks and, consequently, decreased stimulation of insulin and other intestinal peptides (Potter et al. 1981; Bourdon et al. 1999).

Fructans have been studied as prebiotic compounds because they are the ideal substrate for the colonic fermentation of lactic bacteria and the subsequent production of short-chain fatty acids that can have numerous benefits for the host (Roberfroid, 1998). However, there is no clear evidence that the intake of tolerable amounts of this special variety of soluble fibre has any appreciable effect on the lipid profile or glucose metabolism (Ros, 2003a).

\section{Importance of fibre in nuts for cardiovascular health}

As reviewed by Sabaté \& Kelly (2006) in this supplement and elsewhere (Kris-Etherton et al. 2001; Mukuddem-Petersen et al. 2005), the results of observational studies in large cohorts have consistently shown a negative and dose-dependent association between frequent nut intake and the risk for CHD or the risk for cardiovascular or total mortality. 


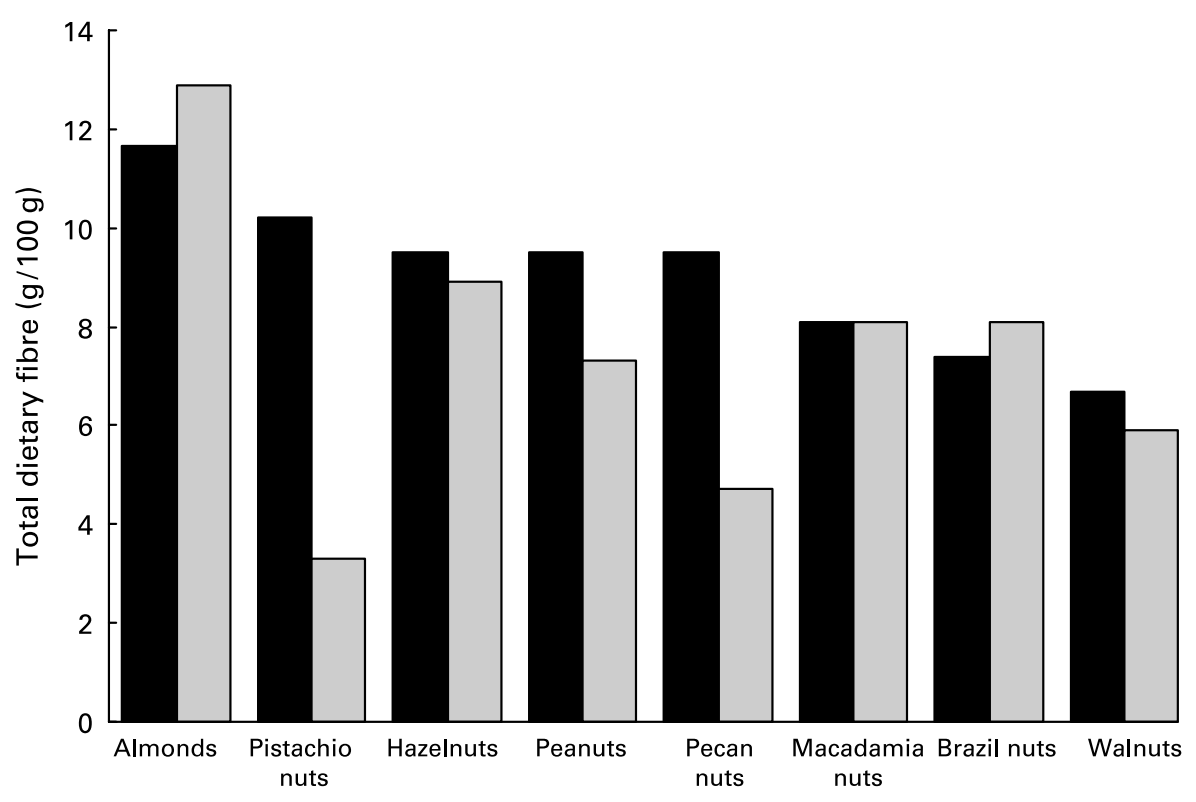

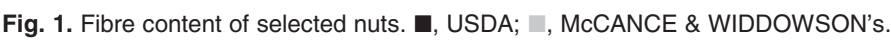

The results of these studies suggests that regular nut consumption reduces the risk of cardiovascular disease by between 30 and $60 \%$, an effect that has been observed in several population groups and is independent of other lifestyle factors. On the other hand, as reviewed by Griel \& Kris-Etherton (2006) in this supplement, clinical studies have consistently shown that regular nut intake improves the lipid profile by reducing total and LDL cholesterol in both normocholesterolaemic and hypercholesterolaemic subjects (Kris-Etherton et al. 2001). The cholesterol-lowering effect of nut intake is greater than that estimated from fatty acid exchange, suggesting that bioactive components other than fatty acids, such as fibre, may be involved (Kris-Etherton et al. 1999).

Epidemiological observations of large cohorts have provided strong evidence of an inverse relationship between the consumption of total dietary fibre and the incidence of cardiovascular diseases, particularly CHD (Lupton \& Turner, 2003; Pereira et al. 2004). The aggregate results of eleven epidemiological studies suggest a mean decrease in

$\begin{array}{cc}\text { Soluble } & \text { Insoluble } \\ \text { (pectin, guar) } & \text { (bran) }\end{array}$

Increased fecal bulk

$\begin{array}{cc}+/- & + \\ & \\ + & - \\ + & - \\ + & -\end{array}$

Glycemic control

$\downarrow$ gastric emptying
$\downarrow$ glucose absorption rate
$\downarrow$ postprandial insulin

$\downarrow$ postprandial insulin
CHD risk of $17 \%$ for every $10 \mathrm{~g}$ fibre added to the diet (Liu et al. 2002). Curiously, most studies have observed a stronger association with cardiovascular protection of insoluble cereal fibre than soluble vegetable or fruit fibre. The results of these and other prospective studies on the incidence of diabetes also consistently reveal that the intake of cereal fibre (or wholegrain cereals) has a protective effect (Montonen et al. 2003). However, the only randomized clinical study that investigated the cardioprotective potential of dietary fibre in patients with a history of CHD failed to find any benefit (Burr et al. 1989). It should be pointed out, however, that in this study wheat bran, and not wholegrain cereal was administered.

Here there is a dilemma: the intake of wholegrain cereals, a source of bran (metabolically inert insoluble fibre), is associated with protection against CHD and diabetes, but there is no evidence that a similar association exists with soluble fibre despite its positive metabolic effects. The reason for this paradox probably lies in the unique composition of wholegrain foods, because plant cell walls are complex matrices containing many bioactive phytochemicals (Milner \& Craig, 2000). Besides bran in the coarse outer layer and as opposed to refined cereal products, whole grains keep the inner embryo layer or germ. Both the bran and the germ are rich in minerals, vitamins, antioxidants and other compounds likely to have significant health benefits. These bioactive food components abound in both the skin and the germ of all seeds (cereals, legumes, nuts), where, among other functions, they serve to protect the plant's DNA from oxidation stress and thus preserve the potential of perpetuating the species. This probably explains why the frequent intake of all types of edible seeds (whole grains, nuts and legumes) and consumption of plant-based diets in general is consistently associated with prevention of cardiovascular diseases in observational studies (Hu, 2003).

Because all kinds of fibre are found in vegetable products, which also contain other physiologically active molecules

Fig. 2. Mechanisms of the metabolic effects of dietary fibre. 
that have beneficial effects on health, a specific advice about fibre is not as important as a recommendation to follow plant-based diets, eating a variety of vegetable products in as natural a state as possible. In the case of cereals, the habitual process of refining leads to important losses of both fibre and phytochemicals, thus consumption of wholegrain cereals should be encouraged.

The available epidemiological evidence shows that fibre in wholegrain cereals and other vegetable products commonly consumed is protective against cardiovascular diseases (Liu et al. 2002; Lupton \& Turner, 2003; Montonen et al. 2003; Pereira et al. 2004). It is unknown, however, whether fibre in nuts also contributes a cardio protective effect. This is because most studies do not correctly identify the intake of this food group. Alternatively, nut consumption is so infrequent that it is difficult to observe differences within the population studied.

Besides the putative health benefits of phytochemicals associated with fibre intake, the protective cardiovascular effect of fibre proper can be explained in several ways. The intake of total fibre can affect satiety and reduce body weight increases over time. Soluble fibre has an additional beneficial effect on lipids and insulin sensitivity. The effects of fibre on body weight, the lipid profile and diabetes are discussed below.

\section{Nuts, fibre and body weight}

The common perception that fatty foods provide excess energy and thus promote obesity has had a negative effect on the image of nuts. However, as reviewed by Rajaram \& Sabaté (2006) in this supplement and elsewhere (Garcia-Lorda et al. 2003; St-Onge, 2005), there is considerable scientific evidence strongly suggesting that frequent nut intake is not associated with weight gain. First, there are epidemiological studies showing a neutral or even negative association between nut intake and BMI. Second, interventional trials in free-living individuals have shown no weight gain or a tendency to lose weight in those assigned to nut diets compared with control diets. Finally, the addition of nuts to low-calorie diets for weight loss results in superior long-term participation and adherence, with consequent improvements in weight loss. Explanations for the lack of a weight-promoting effect of nut intake range from an increased thermogenic effect to increased satiation or incomplete intestinal absorption (Burr et al. 1989; Garcia-Lorda et al. 2003). The latter two effects may be due largely to their high fibre content.

In fact, there is considerable epidemiological evidence that associates the intake of fibre with the regulation of body weight. Obesity is rare in developing countries where large quantities of fibre are consumed. In developed countries, however, where the prevalence of obesity is increasing alarmingly, the trend is to consume less and less complex carbohydrates and fibre. Moreover, vegetarian populations have a lower prevalence of obesity, which suggests that their high fibre intake could play an important role in the prevention of obesity (Newby et al. 2005). Cross-sectional studies show that the intake of both total fibre and soluble fibre is negatively related to total fat intake, body fat content, and BMI, after adjustment for confounding variables. Thus, obese subjects typically consume less fibre than normal weight subjects (Alfieri et al. 1995). Data from a recent cross-sectional study in adolescents also suggest that the intake of wholegrain cereals is associated with a lower BMI (Steffen et al. 2003). In a 10-year prospective study in young adults, fibre intake was associated with lower weight increases and a lower risk of developing obesity after adjusting for the possible confounding effect of fat intake (Ludwig et al. 1999).

Fibre can affect energy balance and, as a result, body weight through a variety of mechanisms that lead to both decreased energy intake and increased energy losses. It has been shown that the intake of sufficient amounts of fibre reduces the sensation of hunger. In fact, the presence of fibre in the diet effectively decreases its energy density, and short-term studies show that a lower energy density increases satiety and decreases intake (Bell \& Rolls, 2001). Additionally, high-fibre foods require greater mastication, which can contribute to the feeling of satiety because the speed of intake is reduced. Likewise, the intake of viscous fibre or foods that are rich in this sort of fibre forms a gel that increases gastric distension and reduces the rate at which the stomach is emptied. This mechanism has been proposed as an explanation for the sensation of fullness and increase in satiety after viscous fibre intake (Jenkins et al. 2000). In turn, soluble fibre decreases the postprandial secretion of insulin (Potter et al. 1981), which may contribute to satiety.

Soluble fibre can also delay or reduce the intestinal digestion and absorption of macronutrients, thus increasing faecal energy losses. In fact, there is a negative relationship between fibre intake and the digestibility of fats and proteins (Howarth et al. 2001). Finally, fibre may also affect the energy balance through its effects on release of intestinal hormones, such as Glucagon-Like Peptide 1, which favours satiety and weight loss, although the mechanisms are only theoretical (Bourdon et al. 1999).

Several intervention studies have been carried out in an attempt to evaluate how enriching the diet with fibre affects body weight. According to a recent review (Howarth et al. 2001), most studies show a decrease in energy intake in response to the consumption of soluble and insoluble fibre supplements (between 6 and $40 \mathrm{~g} / \mathrm{d}$, depending on the studies). The lower energy intake associated with the consumption of fibre leads to a modest reduction in body weight. In this respect, most studies show that high fibre diets (between $5-30 \mathrm{~g} / \mathrm{d}$ ) lead to weight loss, either within the context of ad libitum (Walsh et al. 1984) or energy controlled diets (Ryttig et al. 1989). This extensive review (Howarth et al. 2001) concludes that the intake of approximately $12 \mathrm{~g}$ fibre per day for 4 months in an ad libitum diet is associated with a decrease of $10 \%$ in energy consumption and a weigh loss of approximately $2 \mathrm{~kg}$. These changes are even more favourable in obese patients. A daily portion of $50 \mathrm{~g}$ nuts adds $3-8 \mathrm{~g}$ of fibre to the diet (Fig. 1) and may thus have a beneficial effect on adiposity.

It should be pointed out that some of the studies analyzed were neither randomized nor controlled, were short- or medium- term, or had few participants. Thus it is difficult to draw firm conclusions and the results should be interpreted with caution. What is more, on very few occasions has the degree of adherence to long-term fibre supplement intake been evaluated correctly, although it does not seem to be 
very high because of the unpalatable physical characteristics of the various types of fibre.

\section{Nuts, fibre and insulin sensitivity}

It has been suggested that nuts may improve insulin sensitivity, partly because of their content in fibre and other micronutrients. As Rajaram \& Sabaté (2006) review in this supplement, preliminary evidence from epidemiological studies suggests that frequent nut intake might protect from the development of diabetes. Likewise, feeding trials in diabetic subjects suggest that almonds have a beneficial effect on insulin sensitivity. This may be due in part to the fact that nuts contain mainly unsaturated fatty acids, which have been related to a lower risk of developing diabetes (Meyer et al. 2001) and better glycemic control in diabetic patients (Ros, 2003b). However, an important role is probably played by the high content of fibre and magnesium in nuts, as both nutrients have been associated with a lower risk of developing diabetes (Liu et al. 2002; Lopez-Ridaura et al. 2004). The recent report of an inverse association between nut intake and clinical gallstone disease (Tsai et al. 2004) could also be explained in part by improved insulin sensitivity.

One of the principal goals of medical nutrition therapy for diabetic subjects is to attain and maintain optimal metabolic control of blood glucose, both in the fasting and postprandial state. Among the medical nutrition strategies that lessen postprandial glucose peaks in diabetic subjects are diets that are high in fibre and foods that have a low glycemic index (Wolever et al. 1992), which possibly act by slowing down the absorption of carbohydrates (Jenkins et al. 2000). Population studies have shown that these kind of high-fibre diets may play a protective role in the prevention of type-2 diabetes (Salmeron et al. 1997; Montonen et al. 2003) and cardiovascular diseases (Rimm et al. 1996). Diets that are high in complex carbohydrates and fibre are associated with greater insulin sensitivity and a reduction in plasma insulin levels. It has also been observed that highfibre diets contribute to a better metabolic control in diabetic subjects (Chandalia et al. 2000).

However, in clinical studies it appears that only the viscous variety of soluble fibre plays a significant role in reducing postprandial glycemia (Würsch \& Pi-Sunyer, 1997) and improving other cardiovascular risk factors (Brown et al. 1999). At any rate, there is no direct evidence showing that the intake of fibre from nuts improves insulin sensitivity or the metabolic control of diabetes.

\section{Nuts, fibre and lipid metabolism}

At present, it is clear that dietary factors influence cardiovascular risk largely because of the effect they have on blood cholesterol. Diets that are low in saturated fatty acids and cholesterol and weight loss in obese subjects are the most important dietary-nutritional factors showing a beneficial effect on the lipid profile.

As far as dietary fibre is concerned, numerous observational studies have shown an inverse relation between total fibre intake and cardiovascular and total mortality (Lupton \& Turner, 2003; Pereira et al. 2004). Also, comparative studies between vegetarians and non-vegetarians have shown lower cholesterol levels and mortality rates due to cardiovascular diseases in the former, although it has not been possible to determine whether this is due to the fact that vegetarians consume greater amounts of dietary fibre or is simply a reflection of a greater intake of complex carbohydrates and a lower intake of saturated fatty acids (Sacks et al. 1975).

As discussed, soluble fibre has a salutary effect on lipid metabolism because it increases intestinal viscosity, therefore reducing bile acid absorption and promoting cholesterol catabolism. In particular, a high fibre intake is associated with a decrease in the serum concentrations of total and LDL cholesterol (Jenkins et al. 1993; Brown et al. 1999) and, at the same time, with a lower incidence of coronary disease (Bingham et al. 2003). On the basis of this level of evidence, physicians tend to use high-fibre diets and fibre supplements to decrease blood cholesterol and prevent cardiovascular diseases. Because of their high content in fibre and other healthy compounds, nuts are ideal for this purpose. A recent study in hypercholesterolemic subjects shows how a diet that contains a variety of healthy vegetable foods, including nuts and viscous fibre supplements, can have a notable hypocholesterolemic effect, similar to that of medium doses of statins, the powerful hypolipidemic drugs used for treating hypercholesterolemia (Jenkins et al. 2003). Indeed, part of the decrease in blood cholesterol and cardiovascular risk associated with nut intake may be ascribed to their high fibre content (Kris-Etherton et al. 2001).

\section{Current fibre intake and recommendations}

The consumption of fibre in developed countries has progressively decreased in recent decades. In general, a Western-type diet provides a low fibre intake, largely because of the customary refining of cereals used in bread-making and the production of so many starch-rich foods. Decreased fibre intake in developed countries is also due to the reduction in recent decades of the consumption of bread, potatoes and legumes, which has not been compensated for by an increase in the consumption of fruit and vegetables.

Nutritional data from the European Prospective Investigation into Cancer and Nutrition (EPIC), obtained between 1992 and 1998 in eight large European cohorts, show a mean fibre intake of $23 \mathrm{~g} / \mathrm{d}$ in men and $21.5 \mathrm{~g} / \mathrm{d}$ in women, with the highest intakes in Spanish men and women and the lowest intakes in British men and Swedish women (Bingham et al. 2003). Slightly lower fibre intakes were observed by our group in a longitudinal study of food and nutrient intake carried out in a Spanish Mediterranean population between 1983 and 1993 (Arija et al. 1996). In this 10-year period, fibre intake increased by $6.2 \%$ in men (from 21.8 to $22.3 \mathrm{~g}$ ) and by $13.7 \%$ in women (from 17.5 to $19.9 \mathrm{~g}$ ). It should be pointed out that increased fibre intake was observed in adults, but not in adolescents (Arija et al. 1996).

Current recommendations on fibre intake necessary to maintain good health, proposed by the US Dietary Reference Intake (Food and Nutrition Board, 2002), are $38 \mathrm{~g} / \mathrm{d}$ for men and $25 \mathrm{~g} /$ $\mathrm{d}$ for women (adults below age 50 years). These figures are compatible with recent recommendations from various scientific societies, which propose amounts of 25 and $35 \mathrm{~g} / \mathrm{d}$ (between 10 and $13 \mathrm{~g}$ per $1000 \mathrm{kcal}$ ). Therefore, a $2000 \mathrm{kcal}-$ diet should contain approximately $25 \mathrm{~g}$ of fibre per day, and a diet of $2500 \mathrm{kcal}$, approximately $30 \mathrm{~g}$. The recommended 
insoluble/soluble fibre ratio when the diet is varied and contains wholegrain cereals, nuts, legumes, fruit and other vegetables is the ratio of 3:1 contained in most edible plants.

\section{Conclusion}

Dietary fibre consists of indigestible carbohydrates and lignin, which are intrinsic to plants and can be found whole in them. Fibre is an important component of a plant-based diet, because there is considerable epidemiological evidence to suggest an inverse relationship between the intake of fibre in the habitual diet and cardiovascular risk. This evidence is consistent, powerful and plausible, be it because fibre protects per se or because it is a marker of a healthy dietary pattern. Edible plants mainly contain insoluble fibre, which has no metabolic effects; the minority component, soluble fibre and, particularly, viscous fibre, gelifies intestinal contents, thereby delaying the absorption of glucose and bile acids, and has beneficial effects on the lipid profile and glucose metabolism. However, fibre intake seems to protect against cardiovascular diseases and diabetes independently of the type of fibre and its metabolic effects. This is probably due to the fact that fibre of any sort is associated with the consumption of a wide variety of vegetable products which, as well as fibre, contain numerous bioactive compounds (i.e. antioxidants) with various beneficial effects on health. The vegetables that contain most fibre are wholegrain cereals, followed by nuts. Therefore, to comply with the present recommendations for daily fibre intake and maintain a good cardiovascular and general health, frequent nut consumption is advisable.

\section{Acknowledgements}

This study was partly supported by grants from the Instituto de Salud Carlos III (PI051839), Red de Centros RCMN (C03/08), and Red de Grupos (G03/140) and Ministerio de Educación y Ciencia (AGL22005-03 605/ALI), Madrid, Spain.

\section{References}

Alfieri MAH, Pomerleau J, Grace DM \& Anderson L (1995) Fiber intake of normal weight, moderately obese and severely obese subjects. Obes Res 3, 541-547.

Anderson JW, Allgood LD, Lawrence A, Altringer LA, Jerdack GR, Hengehold DA \& Morel JG (2000) Cholesterol-lowering effect of psyllium intake adjunctive to diet therapy in men and women with hypercholesterolemia: meta-analysis of 8 controlled trials. Am J Clin Nutr 71, 472-479.

Arija V, Salas-Salvadó J, Fernández Ballart J, Cucó G \& Martí-Henneberg C (1996) Consumo, hábitos alimentarios y estado nutricional de la población de Reus (VIII). Evolución de la ingestión de energía y nutrientes entre 1983 y 1993. Med Clin (Barc) 106, 45-50.

Bell EA \& Rolls BJ (2001) Energy density of foods affects energy intake across multiple levels of fat content in lean and obese women. Am J Clin Nutr 73, 1010-1018.

Bingham SA, Day NE, Luben R, et al. (2003) Dietary fibre in food and protection against colorectal cancer in the European Prospective Investigation into Cancer and Nutrition (EPIC): an observational study. Lancet 361, 1496-1501.

Bourdon I, Yokoyama W, Davis P, Hudson C, Backus R, Richter D, Knuckles B \& Scheeman BO (1999) Postprandial lipid, glucose, insulin, and cholecystokinin responses in men fed barley pasta enriched with beta-glucan. Am J Clin Nutr 69, 55-63.

Brown L, Rosner B, Willet WW \& Sacks FM (1999) Cholesterollowering effects of dietary fiber: a meta-analysis. Am J Clin Nutr 69, 30-42.

Burkitt DP, Walker ARP \& Painter NS (1974) Dietary fiber and disease. JAMA 229, 1068-1074.

Burr ML, Fehily AM, Gilbert JF, Rogers S, Holliday RM, Sweetnam PM, Elwood PC \& Deadman NM (1989) Effects of changes in fat, fish, and fibre intakes on death and myocardial reinfarction: diet and reinfarction trial (DART). Lancet 2, 757-761.

Chandalia M, Garg A, Lutjohann D, von Bergmann K, Grundy SM \& Brinkley LJ (2000) Beneficial effects of high dietary fiber intake in patients with type 2 diabetes mellitus. New Engl J Med 342, $1392-1398$.

Food and Nutrition Board (FNB), Institute of Medicine (IOM) (2002) Dietary Reference Intakes for Energy, Carbohydrate, Fiber, Fat, Fatty Acids, Cholesterol, Protein, and Amino Acids (Macronutrients), In: http://books.nap.edu/books/0309085373/html

Garcia-Lorda P, Megias Rangil I \& Salas-Salvado J (2003) Nut consumption, body weight and insulin resistance. Eur J Clin Nutr 57, S8-S11.

Griel AE \& Kris-Etherton PM (2006) Tree Nuts and the Lipid Profile: A Review of Clinical Studies. Br J Nutr 96, Suppl. 2, S68-S78.

Holland B, Welch AA, Unwin ID, Buss DH, Paul AA \& Southgate DAT (1991) Mc'Cance and Widdowson's The Composition of Foods, 5th ed., Cambridge: Royal Society of Chemistry.

Howarth NC, Saltzman E \& Roberts SB (2001) Dietary fiber and weight regulation. Nutr Rev 59, 129-139.

Hu FB (2003) Plant-based foods and prevention of cardiovascular disease: an overview. Am J Clin Nutr 78, Suppl., 544S-551S.

Hu FB \& Willett WC (2002) Optimal diets for prevention of coronary heart disease. JAMA 288, 2569-2578.

Jenkins DJ, Goff DV, Leeds AR, Alberti KG, Wolever TM, Gassull MA \& Hockaday TD (1976) Unabsorbable carbohydrates and diabetes: decreased post-prandial hyperglycaemia. Lancet 2, $172-174$.

Jenkins DJ, Kendall CW, Marchie A, et al. (2003) Effects of a dietary portfolio of cholesterol-lowering foods vs lovastatin on serum lipids and C-reactive protein. JAMA 290, 502-510.

Jenkins DJ, Wolever TM, Rao AV, et al. (1993) Effect on blood lipids of very high intakes of fiber in diets low saturated fat and cholesterol. N Engl J Med 239, 21-26.

Jenkins DJA, Kendall CWC, Axelsen M, Augustin LSA \& Vuksan V (2000) Viscous and nonviscous fibres, nonabsorbable and low glycaemic index carbohydrates, blood lipids and coronary heart disease. Curr Opin Lipidol 11, 49-56.

Kelly JH \& Sabaté J (2006) Nuts and Coronary Heart Disease: an epidemiological perspective. Br J Nutr 96, Suppl. 2, S61-S67.

Kris-Etherton PM, Yu-Poth S, Sabate J, Ratcliffe HE, Zhao G \& Etherton TD (1999) Nuts and their bioactive constituents: effects on serum lipids and other factors that affect disease risk. Am J Clin Nutr 70, 504S-511S.

Kris-Etherton PM, Zhao G, Binkoski AE, Coval SM \& Etherton TD (2001) The effects of nuts on coronary heart disease risk. Nutr Rev 59, $103-111$.

Liu S, Buring JE, Sesso HD, Rimm EB, Willett WC \& Manson JE (2002) A prospective study of dietary fiber intake and risk of cardiovascular disease among women. J Am Coll Cardiol 39, $49-56$.

Lopez-Ridaura R, Willett WC, Rimm EB, Liu S, Stampfer MJ, Manson JE \& Hu FB (2004) Magnesium intake and risk of type 2 diabetes in men and women. Diabetes Care 27, 134-140.

Ludwig DS, Pereira MA, Kroenke CH, Hilner JE, Van Horn L, Slattery ML \& Jacobs DR Jr (1999) Dietary fiber, weight gain, 
and cardiovascular disease risk factors in young adults. JAMA 282, $1539-1546$.

Lupton JR \& Turner ND (2003) Dietary fiber and coronary disease: does the evidence support an association? Curr Atheroscler Rep 5, 500-505.

Marlett JA (1992) Content and composition of dietary fiber in 117 frequently consumed foods. J Am Diet Assoc 92, 175-186.

Meyer KA, Kushi LH, Jacobs DR \& Folsom AR (2001) Dietary fat and incidence of type 2 diabetes mellitus in older Iowa women. Diabetes Care 24, 1528-1535.

Milner JA, Craig L, eds. (2000) Physiologically active food components: their role in optimizing health and aging. Am J Clin Nutr 71, Suppl., 1653S-1743S.

Montonen J, Knekt P, Järvinen R, Aromaa A \& Reunanen A (2003) Whole-grain and fiber intake and the incidence of type 2 diabetes. Am J Clin Nutr 77, 622-629.

Mukuddem-Petersen J, Oosthuizen W \& Jerling JC (2005) A systematic review of the effects of nuts on blood lipid profiles in humans. J Nutr 135, 2082-2089.

Newby PK, Tucker KL \& Wolk A (2005) Risk of overweight and obesity among semivegetarian, lactovegetarian, and vegan women. Am J Clin Nutr 81, 6, 1267-1274.

Parks EJ \& Hellerstein MK (2000) Carbohydrate-induced hypertriacylglycerolemia: historical perspective and review of biological mechanisms. Am J Clin Nutr 7, 412-433.

Pereira MA, O'Reilly E, Augustsson K, et al. (2004) Dietary fiber and risk of coronary heart disease. Arch Intern Med 164, 370-376.

Potter JG, Coffman KP, Reid RL, Krall JM \& Albrink MJ (1981) Effect of test meals of varying dietary fiber content on plasma insulin and glucose response. Am J Clin Nutr 34, 328-334.

Rajaram S \& Sabaté J (2006) Nuts, Body Weight, and Insulin Resistance. Br J Nutr 96, Suppl. 2, S79-S86.

Reaven GM (1997) Do high-carbohydrate diets prevent the development or attenuate the manifestations (or both) of syndrome X? A viewpoint strongly against. Curr Opin Lipidol 8, $23-27$.

Rimm EB, Ascherio A, Giovannucci E, Spiegelman D, Stampfer MJ \& Willett WC (1996) Vegetable, fruit and cereal fiber intake and coronary heart disease among men. JAMA 275, 447-451.
Roberfroid MB \& Delzenne NM (1998) Dietary fructans. Annu Rev Nutr 18, 117-143.

Ros E (2003a) Dietary cis-monounsaturated fatty acids and metabolic control in type 2 diabetes. Am J Clin Nutr 78, $617 \mathrm{~S}-625 \mathrm{~S}$.

Ros E (2003b) Prebiotics and probiotics in the regulation of lipid metabolism. Gastroenterol Hepatol 26, Suppl. 1, 31-36.

Ryttig KR, Tellnes G, Haegh L, Boe E \& Fagerthun H (1989) A dietary fibre supplement and weight maintenance after weight reduction: a randomized, double-blind, placebo-controlled longterm trial. Int $J$ Obes 13, 165-171.

Sacks FM, Castelli WP, Donner A \& Kass EH (1975) Plasma lipids and lipoproteins in vegetarians and controls. N Eng J Med 292, $1148-1151$.

Salmeron J, Manson JE, Stampfer MJ, Colditz GA, Wing AL \& Willett WC (1997) Dietary fiber, glycemic load, and risk of noninsulin-dependent diabetes mellitus in women. JAMA 277, $472-477$.

Steffen LM, Jacobs DR Jr, Murtaugh MA, Moran A, Steinberger J, Hong CP \& Sinaiko AR (2003) Whole grain intake is associated with lower body mass and greater insulin sensitivity among adolescents. Am J Epidemiol 158, 243-250.

St-Onge MP (2005) Dietary fats, teas, dairy, and nuts: potential functional foods for weight control? Am J Clin Nutr 81, 7-15.

Tsai CJ, Leitzmann MF, Hu FB, Willett WC \& Giovannucci EL (2004) Frequent nut consumption and decreased risk of cholecystectomy in women. Am J Clin Nutr 80, 76-81.

U.S. Department of Agriculture, Agricultural Research Service. 1984. Composition of foods: Nut and seed products; raw, processed, prepared. Agriculture Handbook 8-12

Walsh DE, Yaghoubian V \& Behforooz A (1984) Effect of glucomannan on obese patients: a clinical study. Int $J$ Obes $\mathbf{8}$, 289-293.

Wolever TM, Jenkins DJA, Vuksan V, Jenkins AL, Wong GS \& Josse GS (1992) Beneficial effect of low-glycemic index diet in overweight NIDDM subjects. Diabetes Care 15, 562-564.

Würsch P \& Pi-Sunyer FX (1997) The role of viscous soluble fiber in the metabolic control of diabetes. Diabetes Care 20, $1774-1780$. 